\title{
Investigations of hollow-core photonic crystal fibres (HC-PCF) for trace explosive vapour detection.
}

\author{
JOHNY, J., KARNIK, S. and PRABHU, R.
}

(C) 2020 Society of Photo-Optical Instrumentation Engineers (SPIE). One print or electronic copy may be made for personal use only. Systematic reproduction and distribution, duplication of any material in this publication for a fee or for commercial purposes, and modification of the contents of the publication are prohibited. 


\title{
Investigations on Hollow Core Photonic Crystal Fibres (HC-PCF) for trace explosive vapour detection

\author{
Jincy Johny, Shruti Karnik, Radhakrishna Prabhu \\ School of Engineering, Robert Gordon University, Garthdee Road, Aberdeen, UK, AB10 7GJ
}

\begin{abstract}
Trace detection and identification of hazardous volatile explosives has been a key challenge to the scientific community past many decades. Commercially available various analytical and spectroscopic techniques suffer from low sensitivity, swabbing of surfaces and low detection limit. Triacetone Triperoxide (TATP), used in improvised explosive devices IEDs vaporizes readily at room temperature and has a vapour signature. However, explosive trace detectors (ETDs) are incompetent to detect TATP due to absence of chromophoric groups. We have investigated the novel hollow core photonic crystal fibres (HC-PCFs) based Raman sensor for real time monitoring of such volatile explosives in airport security. Raman scattering, a powerful, non-destructive tool provides molecular fingerprinting and is a potential candidate for detection of trace explosives but suffer from weak signal strength. Simultaneous confinement of pumped light and gas in HCFs allows greater light gas interaction providing an excellent optical sensing platform. These sensors can be easily incorporated at the security terminals or baggage counters with the existing metal detection systems. This paper reports investigations carried out on the HC-PCF designed using COMSOL Multiphysics software. The Raman signal being dependent on its intensity and mode area, simulations were conducted to analyse PCF parameters like confinement losses and mode field diameter/ effective mode area and its associated wavelength dependency. Theoretical study carried out on the HC-PCF also revealed that mode field confinements within the hollow core can be modified to suit specific laser wavelengths and confinement losses can be reduced to achieve Raman signal enhancement by optimizing their geometrical parameters like air-hole size and pitch/ hole-to-hole distance.
\end{abstract}

Keywords: Hollow core photonic crystal fibres (HC-PCF), trace vapor detection, TATP, Improvised explosive devices IEDs, Raman scattering, computational modelling, mode field diameter, mode field confinement

\section{INTRODUCTION}

Improvised explosive devices (IEDs) are homemade destructive bombs/ devices that have been employed in many bombings including 2015 Paris attacks, the 2016 Brussels Airport bombings, the 2017 concert bombing in Manchester UK, and recently the 2018 bombings in Surabaya, Indonesia. Peroxide based IEDs utilize Triacetone Triperoxide (TATP), DADP, HMTD and TNT as high-powered explosive ${ }^{1}$. TATP and DADP, cyclic peroxides prepared from easily available chemicals such as acetone and peroxide, are extremely sensitive to shock, friction, abrasion and static electricity ${ }^{2}$. Difficulty in handling and storage of TATP has been the obvious reason it is not used for commercial and military purposes ${ }^{3}$. The explosive power of TATP which is comparable to TNT and it has a characteristic chemical vapor signature. Since TATP and DADP does not possess any chromophoric group (example, nitro explosives such as RDX, TNT, PETN), metallic elements and aromaticity, general explosive trace detectors ETDs or fluorescent detectors are unable to detect them. Several sophisticated analytical techniques used for trace vapor detection are, solid phase extraction and liquid chromatography mass spectrometry, Dielectric Barrier Discharge Ionization-Mass Spectrometry (DBDIMS), ultraviolet femtosecond multiphoton ionization time-of-flight mass spectrometry, Ion mobility spectrometry (IMS) and metal oxide based conductometric sensors ${ }^{1,4-8}$. Chromatographic and spectrometric techniques require swabbing of surfaces, conductometric sensors have poor selectivity, whereas other techniques either require installation of bulky instrumentation or are time consuming and cannot be employed in fast moving venues. Furthermore, use of canines is another potential way TATP can be detected, however, the logistics, expensive costs for maintaining and training the dogs and handlers are probable issues encountered. Thus, no-swab sniffer techniques with nanosecond screening, high sensitivity and easy installation are desired at fast moving and high security venues such as airports and places of national security. 
Raman spectroscopy is an excellent technique for detection of various chemical entities based on their molecular structure and each molecule has a unique chemical signature ${ }^{9}$. Thus, molecular fingerprinting achieved by Raman scattering has opened avenues for its utilization in gas sensing platforms. Weak Raman scattering and low particle densities of the gases in the free space result in poor sensitivity ${ }^{9,10}$. Enhancement in Raman signal can be achieved by employing hollow core photonic crystal fibres that enable strong light-gas interaction over extended path length. HCF-Raman sensor is devised as an absorption cell with laser pumped from one end and travels throughout the HCF to the detector end. This system requires low analyte volume and fast response time and thus highly sensitive for trace volatile explosive detection. Thus, HC-PCF based Raman sensing for detection of trace volatile explosives, would allow fast remote sensing with much lower detection limits (ppm level). Hollow core photonic crystal fibres already have epitomized internet and telecommunication applications. HC-PCFs have great advantage in the field of optical sensing because of their compactness, remote sensing ability, multiplexing capability, rapid response time, sensitivity, electromagnetic compatibility freedom from corrosion and ability to operate in extreme environmental conditions ${ }^{11}$. They are often more flexible than normal optical fibres, as it is possible to manage their properties, leading to a freedom of design which is essential for sensing. HC-PCFs have low transmission efficiency and low bending losses due to the air core and enhanced sensitivity. However, interference due to Raman bands originating from the fused silica of the fibre material and fluorescence background can limit the sensitivity of the detection technique ${ }^{10}$. Thus, it is essential to tailor the performance of HC-PCFs as Raman signal is highly dependent on its intensity and mode area. HC-PCFs ${ }^{12,13}$ have specialized geometrical structure (core air hole cladding) and unique properties such as their guiding mechanisms and modal characteristics. Propagation characteristics of PCFs can be tuned by altering different structural or physical parameters like core diameter $(\rho)$, cladding hole diameter $(d)$ and pitch $(\mathrm{p})^{14}$. All these intrinsic properties of PCFs are being exploited for different sensing applications. Furthermore, these Raman based distributed fibre optic sensors achieves signal enhancement due to the length of the fibre as well. In this work, we have optimized HCPCF by varying above geometrical parameters using COMSOL Multiphysics software. This study will enable us to design a suitable HC-PCF based Raman system for sensing trace volatile explosives such as TATP, DADP, HMTD and even DNT, a volatile by-product of TNT. Such a sensing system would require puffer machines to sweep of any traces or vapours of these illicit explosives. This remote sensing sniffing device that is compact and electromagnetically compatible could be easily installed at the security terminal, baggage counters and baggage carousels at airports.

\section{THEORY}

PCFs are optical fibres with a complex refractive index profile that employs a microstructured arrangement of low-index material in a background material of higher refractive index ${ }^{15}$. Normally the background materials used in PCFs are pure or undoped silica and the low index cladding region consists of many number of air voids also known as air holes ${ }^{16}$. Typically, photonic crystals are periodic optical (micro or nano) structures running axially along the length of the optical fibre, that affects the propagation characteristics of the electromagnetic waves travelling through its core $^{17}$. In our past studies, we have reported modelling and simulation studies for FBG/ PCF-FBG sensors for various oil and gas sensing applications ${ }^{18-22}$.

PCFs normally have two modes of operation based on their light guiding technique - index guiding and bandgap guiding ${ }^{23}$. Index guiding PCFs operates like conventional optical fibres wherein light is confined within the high index core by modified total internal reflection (M-TIR) principle ${ }^{24}$. However, bandgap PCFs guides light in the low index core region by the reflection from photonic crystal cladding ${ }^{25}$. In contrast to solid-core PCF, in which guidance is by step-index total internal reflection, in hollow core PCF light is confined due to photonic bandgap that arises from the regular 2-dimensional array of air holes in the cladding ${ }^{26}$. 


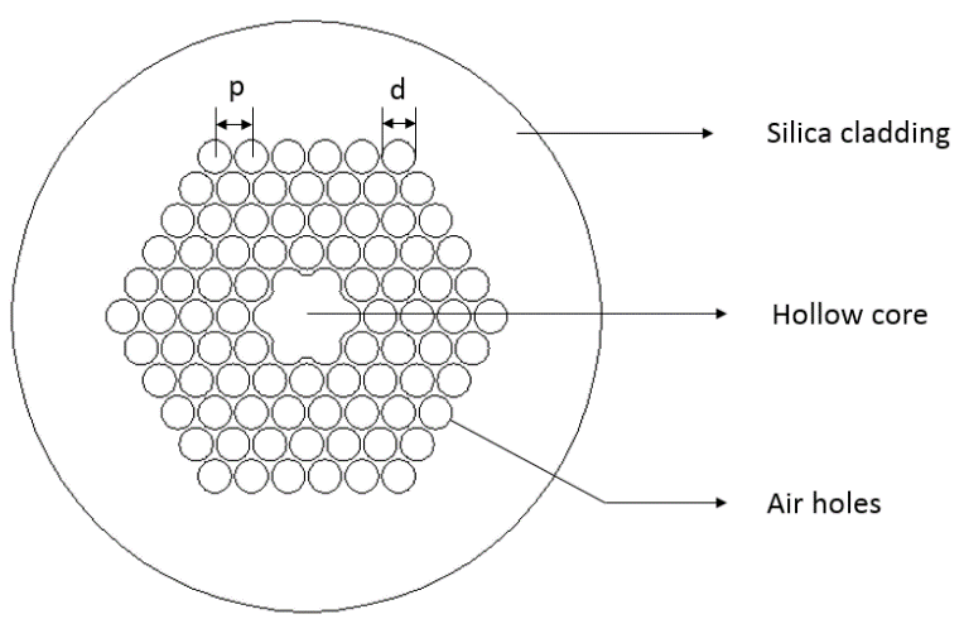

Figure 1: Cross-section of hollow core PCF

Figure 1 shows the cross-sectional view of the designed hollow core PCF with microstructured cladding. Effective mode area and confinement losses are important propagation characteristic of the PCF which can be tuned by altering its physical parameters like core diameter $(\rho)$, cladding hole diameter $(d)$ and pitch $(p)$ in combination with the choice of material refractive index and type of crystal lattice ${ }^{27,28}$. The Raman signal is normally dependent on its signal intensity. Therefore, optimisation of the mode field area and light leakage losses would in turn enhance the Raman signal from the HC-PCF.

Effective mode area $\left(\mathrm{A}_{\mathrm{eff}}\right)$ is considered as the light carrying region of the PCF and is given by the equation ${ }^{29,30}$ :

$$
A_{\text {eff }}=\frac{\left[\iint_{-\infty}^{\infty}|E(x, y)|^{2} d x d y\right]^{2}}{\iint_{-\infty}^{\infty}|E(x, y)|^{4} d x d y}
$$

Where, $\mathrm{E}(\mathrm{x}, \mathrm{y})$ is the electric field distribution.

Also, effective mode area is related to mode field diameter (MFD) by the equation ${ }^{31}$ [26]:

$$
\mathrm{A}_{\mathrm{eff}}=\mathrm{k}_{\mathrm{n}}\left(\frac{\pi}{4}\right) \mathrm{MFD}^{2}=\mathrm{k}_{\mathrm{n}} \pi \mathrm{w}^{2}
$$

where $k_{n}$ is the correction factor and spot size, $\mathrm{w}=\mathrm{MFD} / 2$.

Mode field diameter is approximated as,

$$
\mathrm{MFD} \approx \frac{2}{\sqrt{\pi}} \sqrt{\mathrm{A}_{\mathrm{eff}}}
$$

Confinement or leakage loss $\left(L_{c}\right)$ is the light confinement ability of the PCF within its core and can be calculated from the imaginary part of its effective refractive index [32].

$$
L_{c}=\frac{\left(\frac{\dot{20}}{\ln (10)}(2 \pi)\right) \operatorname{Im}\left(\mathrm{n}_{\mathrm{eff}}\right)}{\lambda}
$$

where $\operatorname{Im}\left(\mathrm{n}_{\mathrm{eff}}\right)$ is the imaginary part of effective refractive index, $\mathrm{n}_{\mathrm{eff}}$ and $\lambda$ is the propagating wavelength. Confinement loss depends on the transmission wavelength, PCF structure, size, shape, number of air holes and number of air hole rings. 


\section{RESULTS AND DISCUSSION}

The HC-PCF was designed using COMSOL Multiphysics software, with an air core (refractive index of air, $n_{\text {air }}=1$ ) and a four ring microstructured cladding of background material silica (refractive index of silica, $n_{s i}=$ 1.45). Simulations were conducted on the designed HC-PCF to analyse fibre propagation characteristics like effective mode area, electric field intensity and its associated wavelength dependency.

Figure 2 (a) and (b) show the electric field profile of the fundamental mode obtained for the designed HC-PCF at $785 \mathrm{~nm}$. The central core with red colour indicates the region of highest electric field. The geometrical parameters, pitch/ hole to hole centre distance, $p=4.64 \mu \mathrm{m}$ and diameter of cladding air holes, $d=4.176 \mu \mathrm{m}$.
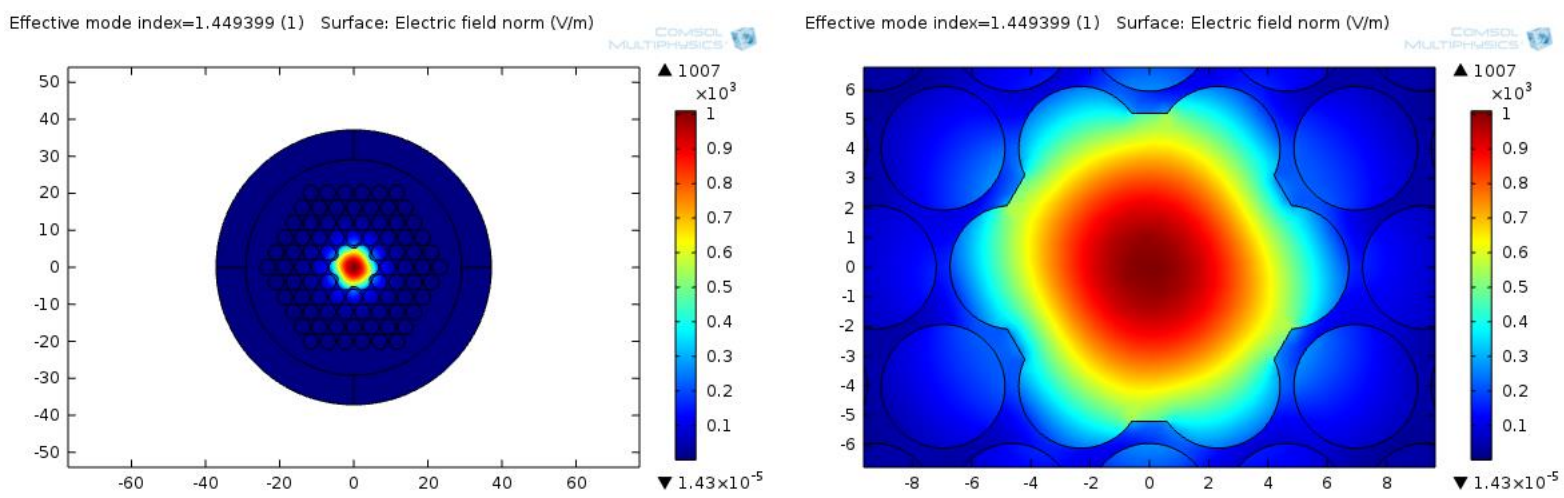

Figure 2: (a) Electric field mode profile of the HC-PCF, (b) Zoomed view of electric field mode profile in HC-PCF core (at $785 \mathrm{~nm}$ )

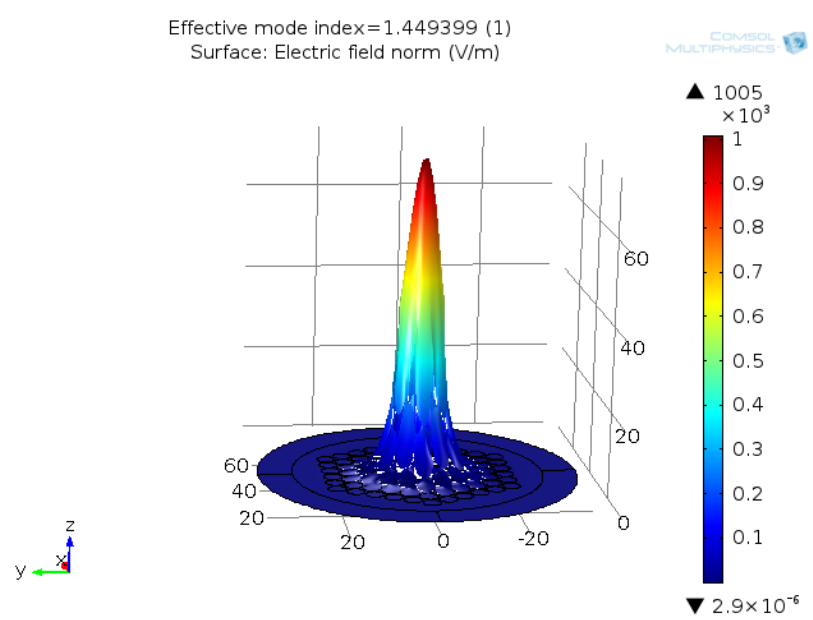

Figure 3: 3D View of electric field mode profile of the HC-PCF (at $785 \mathrm{~nm}$ )

Figure 3 depicts the three-dimensional (3D) view of the Gaussian electric field pattern obtained for the HC-PCF. At $785 \mathrm{~nm}$, the effective mode area obtained for the electric field confinement $=63.8 \mu \mathrm{m}^{2}$ and the corresponding mode field diameter calculated from effective area $=9.01 \mu \mathrm{m}$. 


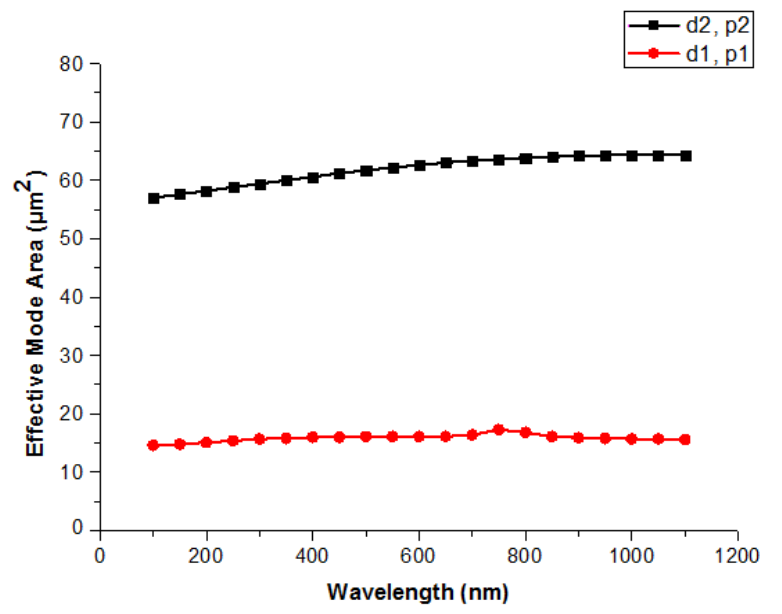

(a)

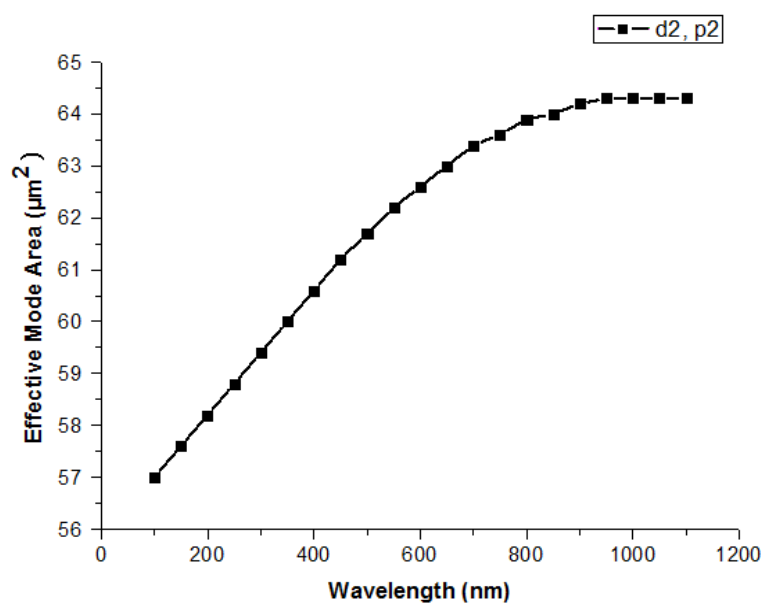

(b)

Figure 4: (a) Effective Mode Area vs Wavelength for $d_{1}=2.088 \mu \mathrm{m}, p_{1}=2.32 \mu \mathrm{m}$ and $d_{2}=$ $4.176 \mu \mathrm{m}, p_{2}=4.64 \mu \mathrm{m}$ (b) Zoomed view of Effective Mode Area vs Wavelength for $d_{2}=4.176 \mu \mathrm{m}, p_{2}=$ $4.64 \mu \mathrm{m}$

Figure 4 (a) shows the variation in effective mode area with respect to wavelength (in $\mathrm{nm}$ ) for the two simulations conducted on the HC-PCF with $d_{1}=2.088 \mu \mathrm{m}, p_{1}=2.32 \mu \mathrm{m}$ and $d_{2}=4.176 \mu \mathrm{m}, p_{2}=4.64 \mu \mathrm{m}$. It can be observed that the effective area increases with increasing values for the HC-PCF geometrical parameters like pitch $(p)$ and cladding air hole diameter $(d)$. From Figure 4 (b) it can be observed that effective mode area increases with wavelength.

Table 1: Electric Field Intensity for various laser wavelengths

\begin{tabular}{|c|c|}
\hline Wavelength (nm) & Electric Field Intensity (V/m) \\
\hline 1064 & $0.772 \times 10^{6}$ \\
\hline 785 & $1.005 \times 10^{6}$ \\
\hline 532 & $1.425 \times 10^{6}$ \\
\hline 355 & $2.040 \times 10^{6}$ \\
\hline 266 & $2.645 \times 10^{6}$ \\
\hline
\end{tabular}

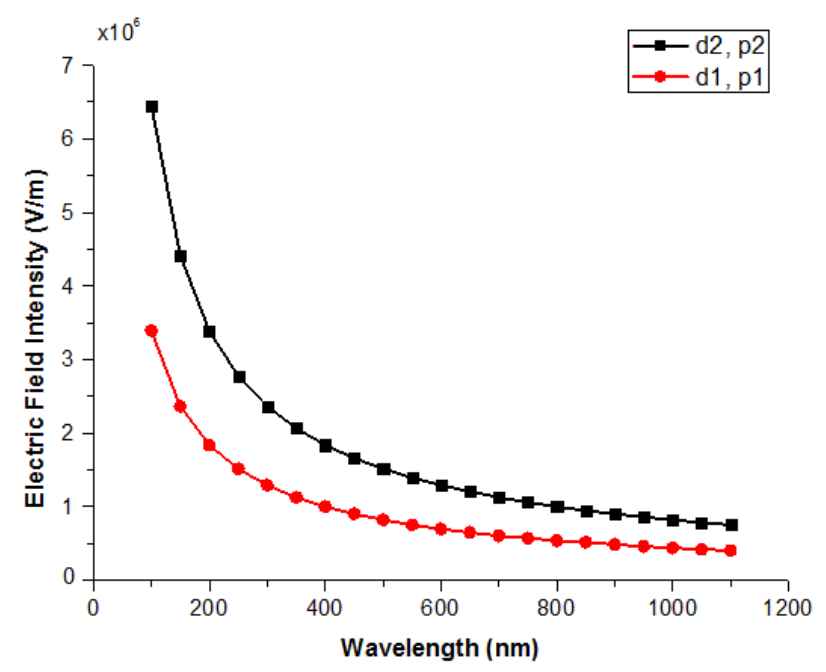

Figure 5: Electric Field Intensity vs Wavelength for $d_{1}=2.088 \mu \mathrm{m}, p_{1}=2.32 \mu \mathrm{m}$ and $d_{2}=4.176 \mu \mathrm{m}, p_{2}=$ $4.64 \mu \mathrm{m}$ 
Theoretical study carried out on the HC-PCF revealed that mode field confinements within the hollow core can be modified to suit specific Raman laser wavelengths by optimizing their geometrical parameters like air-hole size and pitch/ hole-to-hole distance. Table 1 shows the electric field intensity value obtained through simulation for various laser wavelengths.

Figure 5 depicts the changes in electric field intensity with respect to wavelength (in $\mathrm{nm}$ ) for the simulations conducted on the HC-PCF with $d_{1}=2.088 \mu \mathrm{m}, p_{1}=2.32 \mu \mathrm{m}$ and $d_{2}=4.176 \mu \mathrm{m}, p_{2}=4.64 \mu \mathrm{m}$. From the graph shown in Figure 5 it can be observed that electric field intensity decreases with wavelength. Furthermore, the electric field intensity graph also experiences an upward shift with increasing values for cladding air hole diameter $(d)$ and pitch $(p)$. The various simulations conducted on the air core PCF also identified the possibilities of photonic bandgap optimisations through the PCF structural modifications.

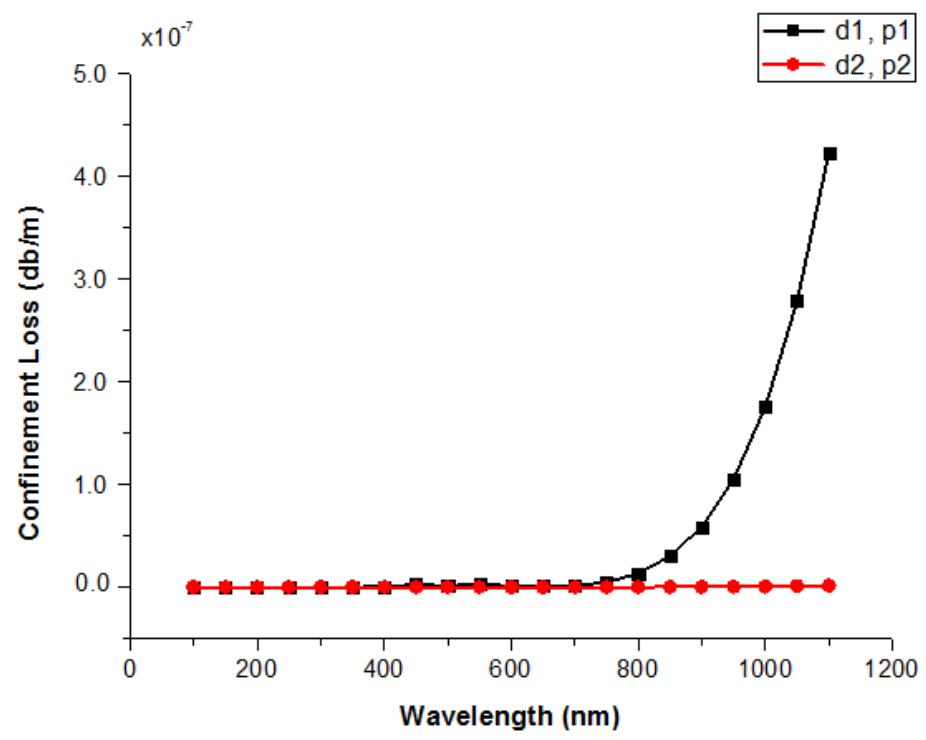

Figure 6: Confinement Loss vs Wavelength for $d_{1}=2.088 \mu \mathrm{m}, p_{1}=2.32 \mu \mathrm{m}$ and $d_{2}=4.176 \mu \mathrm{m}, p_{2}=4.64 \mu \mathrm{m}$

Figure 6 shows the variation in confinement loss with respect to wavelength (in $\mathrm{nm}$ ) for the simulations conducted on the HC-PCF with $d_{1}=2.088 \mu \mathrm{m}, p_{1}=2.32 \mu \mathrm{m}$ and $d_{2}=4.176 \mu \mathrm{m}, p_{2}=4.64 \mu \mathrm{m}$. From the graph shown in Figure 6 we can observe that confinement loss significantly reduces with increasing values for cladding air hole diameter $(d)$ and pitch $(p)$.

The various simulations conducted on the designed HC-PCF identified the possibility of optimising their electric field intensity and confinement losses in order to enhance the Raman signal from the HC-PCF.

\section{CONCLUSION}

The computational study conducted on the HC-PCF designed using COMSOL Multiphysics software helped in analysing key PCF parameters like effective mode area, confinement losses, electric field intensity and its associated wavelength dependency. It was found that the HC-PCF modal properties are a strong function of its structural parameters such as, air hole size and spacing of the microstructured air holes in the cladding. Simulations conducted on the HC-PCF identified that the electric field intensity, effective mode area and leakage losses can be optimised through PCF geometrical modifications which are also important parameters for the Raman signal. Effective mode area and electric field intensity were found increasing with increasing values for air hole size and spacing. The effective mode area showed an enhancing trend with increasing wavelength; however, the electric field intensity showed a reducing trend with increasing wavelength. These studies suggest that the Raman signal from the Raman-HCF based sensor can be improved by proper optimisation of the HC-PCF geometrical parameters. Furthermore, the theoretical study carried out on the HC-PCF also revealed that mode field confinements within the hollow core can be modified to suit specific Raman laser wavelengths by optimizing their 
geometrical parameters like air-hole size and pitch/hole-to-hole distance. Furthermore, simultaneous confinement of laser pumped light and gas in the HC-PCF allows greater light-gas interaction, which in turn enhance their sensing capabilities. These theoretical optimization studies can be exploited in the future designs of highly sensitive remotely operated Raman-HCF based sensing sniffer devices for trace detection of volatile explosives used in IEDs.

\section{REFERENCES}

[1] Rossi, A. S., Ricci, P. and Gregory, O. J., "Trace Detection of Explosives Using Metal Oxide Catalysts," IEEE Sens. J. 19(13), 4773-4780 (2019).

[2] Dubnikova, F., Kosloff, R., Almog, J., Zeiri, Y., Boese, R., Itzhaky, H., Alt, A. and Keinan, E., "Decomposition of triacetone triperoxide is an entropic explosion," J. Am. Chem. Soc. 127(4), 11461159 (2005).

[3] Meyer, R., Köhler, J., Homburg, A., [Explosives 6th Edition], Wiley-VCH Verlag GmbH \& Co. KGaA (2019).

[4] Gamble, S. C., Campos, L. C. and Morgan, R. M., "Detection of trace peroxide explosives in environmental samples using solid phase extraction and liquid chromatography mass spectrometry," Environ. Forensics 18(1), 50-61 (2017).

[5] Hagenhoff, S., Franzke, J. and Hayen, H., "Determination of Peroxide Explosive TATP and Related Compounds by Dielectric Barrier Discharge Ionization-Mass Spectrometry (DBDI-MS)," Anal. Chem. 89(7), 4210-4215 (2017).

[6] Ezoe, R., Imasaka, T. and Imasaka, T., "Determination of triacetone triperoxide using ultraviolet femtosecond multiphoton ionization time-of-flight mass spectrometry," Anal. Chim. Acta 853(1), 508$513(2015)$.

[7] Ewing, R. G., Waltman, M. J. and Atkinson, D. A., "Characterization of triacetone triperoxide by ion mobility spectrometry and mass spectrometry following atmospheric pressure chemical ionization," Anal. Chem. 83(12), 4838-4844 (2011).

[8] Ricci, P. P., Rossi, A. S. and Gregory, O. J., "Orthogonal Sensors for the Trace Detection of Explosives," IEEE Sensors Lett. 3(10), 2-5 (2019).

[9] Long, D. A., [The Raman effect: a unified treatment of the theory of Raman scattering by molecules. 2002] (2002).

[10] Yan, D., Popp, J. and Frosch, T., "Analysis of Fiber-Enhanced Raman Gas Sensing Based on Raman Chemical Imaging," Anal. Chem. 89(22), 12269-12275 (2017).

[11] Lee, B., "Review of the present status of optical fiber sensors," Opt. Fiber Technol. 9(2), 57-79 (2003).

[12] Kirchhof, J., Kobelke, J., Schuster, K., Bartelt, H., Iliew, R., Etrich, C. and Lederer, F., "Photonic crystal fibers," Photonic Cryst. Adv. Des. Fabr. Charact. 106(2), 267-288 (2006).

[13] Knight, J. C., Birks, T. A., Russell, P. S. J. and Atkin, D. M., "All-silica single-mode optical fiber with photonic crystal cladding," Opt. Lett. 21(19), 1547-1549 (1996).

[14] Johny, J., Prabhu, R. and Fung, W. K., "Computational study of nanostructured composite materials for photonic crystal fibre sensors,” \{IOP\} Conf. Ser. Mater. Sci. Eng. 195, 12012 (2017).

[15] Guobin, R., Zhi, W., Shuqin, L., Yan, L. and Shuisheng, J., "Full-vectorial analysis of complex refractiveindex photonic crystal fibers," Opt. Express 12(6), 1126 (2004).

[16] Cucinotta, A., [Photonic crystal fiber: Theory and fabrication], Bentham Science (2012).

[17] Dainese, P., Russell, P. S. J., Joly, N., Knight, J. C., Wiederhecker, G. S., Fragnito, H. L., Laude, V. and Khelif, A., "Stimulated Brillouin scattering from multi-GHz-guided acoustic phonons in nanostructured photonic crystal fibres," Nat. Phys. 2(6), 388-392 (2006).

[18] Johny, J., Summers, J., Bhavsar, K., Joseph, G. P., Fung, W. K. and Prabhu, R., "Theoretical investigation of positional influence of FBG sensors for structural health monitoring of offshore structures," Oceans. 2017 - Aberdeen 2017-October, 1-5, IEEE (2017).

[19] Johny, J., Prabhu, R. and Fung, W. K., "Investigation of structural parameter dependence of confinement losses in PCF-FBG sensor for oil and gas sensing applications,” Opt. Quantum Electron. 48(4), 1-9 (2016).

[20] Johny, J., Prabhu, R., Fung, W. K. and Watson, J., "Investigation of positioning of FBG sensors for smart 
monitoring of oil and gas subsea structures," Ocean. 2016 - Shanghai, 3-7 (2016).

[21] Udoh, S., Njuguma, J. and Prabhu, R., "Modelling and Simulation of Fiber Bragg Grating Characterization for Oil and Gas Sensing Applications," SIMS '14 Proc. 2014 First Int. Conf. Syst. Informatics, Model. Simul. Model., 213-218 (2014).

[22] Amos, S., Prabhu, R. and Njuguna, J., "Theoretical design and analysis of a sensing system for high pressure and temperature measurement in subsea underwater applications.," Oceans. 2017 - Aberdeen, 2680-2686, IEEE (2017).

[23] Pal, B. P., [Guided Wave Optical Components and Devices: Basics, Technology, and Applications], Academic Press (2010).

[24] Wehrspohn RB, Kitzerow H, B. K., [Nanophotonic Materials: Photonic Crystals, Plasmonics, and Metamaterials], John Wiley \& Sons (2008).

[25] Xiao, L., Jin, W. and Demokan, M. S., "Photonic crystal fibers confining light by both index-guiding and bandgap-guiding: hybrid PCFs," Opt. Express 15(24), 15637-15647 (2007).

[26] Lim, H. and Wise, F. W., "Control of dispersion in a femtosecond ytterbium laser by use of hollow-core photonic bandgap fiber,” Opt. Express 12(10), 2231-2235 (2004).

[27] Pinto, A. M. R. and Lopez-Amo, M., "Photonic crystal fibers for sensing applications," J. Sensors 2012 (2012).

[28] Ahmed, K. and Morshed, M., "Design and numerical analysis of microstructured-core octagonal photonic crystal fiber for sensing applications,” Sens. Bio-Sensing Res. 7, 1-6 (2016).

[29] Miyagi, K. and Namihira, Y., "Measurements of mode field diameter and effective area for photonic crystal fibers by far field scanning technique," Opt. Rev. 17(4), 388-392 (2010).

[30] Rifat A.A., Ahmed K., Asaduzzaman S., Paul B.K., A. R., [Development of Photonic Crystal Fiber-Based Gas/Chemical Sensors.], Computational Photonic Sensors, Springer (2019).

[31] Mortensen, N. A., "Effective area of photonic crystal fiber," Opt. Express 10(7), 341-348 (2002). 